\title{
BOVINE MAMMARY GLAND DURING THE DRY PERIOD: 2. CHANGES IN BODY WEIGHT, MORPHOLOGICAL FEATURES FOR MAMMARY GLAND AND QUANTIFICATIONS OF NUCLEIC ACIDS
}

\author{
A.S.M. Soliman ${ }^{1}$, A.I.M. El-Sayed ${ }^{1}$, A.M. El-Gaafarawy ${ }^{2}$ and M.A.E. Ali ${ }^{2}$ \\ 1- Animal Production Department, Faculty of Agriculture, Benha University, 2- \\ Animal Production Research Institute, Agriculture Research Center, Ministry of \\ Agriculture
}

\section{SUMMARY}

This study was carried out to study and relate the changes in cow body weight on dimensions of the mammary gland morphology and estimate mammary cell number and its secretory activity by using multiple biopsies to quantitatively evaluate changes in DNA and RNA content during different stages of dry periods. Sixteen pregnant Friesian cows were divided into two groups (8 cows each). The first included cows with dry period more than 60 days. While the second included cows with dry period less than 45 days. Changes in body weight (kg) cows and mammary gland measurements were estimated weekly. While mammary tissue biopsies were taken from rear quarters once from each cow.

Cows increased by averages of 10.62 and $6.01 \%$ in body weight during the dry period, while they lost 8.50 and $7.14 \%$ at calving for cows having long and short dry period lengths, respectively. Udder volume decreased during the active involution (27.49 and $17.38 \%$ for cows having long and short dry periods, respectively). While during redevelopment and colostrogenesis phase and at 1 st day postpartum it increased significantly for cows having long dry period (167.97 and $90.48 \%$, respectively) and were (47.62\% and $44.97 \%$, respectively) for cows having short dry period. During end of lactation an average concentrations of DNA were 3.25 and $3.15 \mathrm{mg} / \mathrm{g}$ decreased to reach 3.00 and $2.86 \mathrm{mg} / \mathrm{g}$ during active involution for cows will having long and short dry period lengths, respectively. Also, an average concentrations of RNA were 5.71 and $5.91 \mathrm{mg} / \mathrm{g}$ during the end of lactation period decreased to attained 4.22 and $4.80 \mathrm{mg} / \mathrm{g}$ during active involution phase for cows will having long and short dry period lengths, respectively. While during redevelopment and colostrogenesis phase concentrations of RNA increased to 6.01 and $6.26 \mathrm{mg} / \mathrm{g}$ for cows having long and short dry periods, respectively. The ratio of RNA: DNA was greater for cows having short dry period throughout the experimental periods. These obtained data suggest that the value of the dry period might be to repair or replace damaged or senescent mammary epithelial cells prior to the next lactation.

Keywords: cows, dry period, body weight, morphological features for mammary, DNA, RNA

\section{INTRODUCTION}

Physical characteristics of the udder and teats were related to economically important traits in dairy cows. Shape, attachments of the udder, size, shape and placement of the teats are generally considered as important characters for dairy cows.

Issued by The Egyptian Society of Animal Production 
The importance of concomitant pregnancy during the dry period has been addressed by a limited number of investigators. Capuco et al. (1997) assessed mammary gland involution on the basis of udder weight, DNA content, and histology. Zhang et al. (1994) reported that, significant relationship occurs between udder size (suspension, distance from the floor, and shape of the udder and teats) and mastitis incidence. Therefore, selection for decreased somatic cell count, high udder suspension, shorter and more closely located teats, will lead to reduction in mastitis incidence.

This study was carried out to study and relate the changes in cow body weight on dimensions of the mammary gland morphology and quantifications of the nucleic acids at the end of lactation, during the different stages of dry periods and postpartum period.

\section{MATERIALS AND METHODS}

The field study of this experiment was carried out at Sakha Experimental Station, belonged to Animal Production Research Institute, Agriculture Research Center, Ministry of Agriculture, Dokki, Giza, Egypt. Both DNA and RNA of mammary tissue analysis were executed at Molecular Genetic Laboratory, The International Livestock Management Training Center (ILMTC).

\section{Experimental animals:}

A total number of 16 pregnant Friesian cows aged 3.5 to 6.5 years, and had 2 to 4 parities. These cows were divided into two groups ( 8 cows each). The first group included cows with dry period more than 60 days (more than the optimum period). While the second group included cows with dry period less than 45 days (less than optimum period).

\section{Management and feeding:}

Cows were housed loose in semi-shaded open yard throughout the experimental periods and fed on green Berseem (Trifolium alexandrinum) and concentrate mixture during December to end of April. While during November, May and June they fed on corn silage, Berseem hay and concentrate mixture. Also they fed rice straw ad libitum as a roughage allover the year. Feed was offered twice daily at 08:00 $\mathrm{h}$ and 16:00 $\mathrm{h}$. and drinking fresh water was available.

Cows were dried off by using abrupt cessation of milking method, when cow giving 1 to $2 \mathrm{Kg}$ /day. After the last milking, teats of each cow were washed and infused with antibiotics which help it to protect infections that may occur in early involution.

The studied dry periods were divided into three phases; 1) Active involution phase (AI, the beginning of the dry period - 28 days after drying off). 2) Steady state involution phase (SSI, mid-dry period - its length depends on total length of the dry period). 3) Redevelopment and colostrogenesis phase (RC, the last of dry period - 28 days before parturition).

\section{Changes in body weight and morphological features for mammary gland:}

Changes in body weight $(\mathrm{kg})$ of cows were determined weekly after morning milking and before feeding. Mammary gland measurements were taken at weekly intervals, by using of zoometric tools, just after afternoon milking and during dry 
period, as shown in Fig. (1). These measurements of the udder traits were (cm), udder periphery, fore depth, rear depth, fore width, rear width, distance between rear teats, distance between fore teats, distance between fore and hind teats, length and diameter of fore and rear teats, distance of the udder from the floor. Udder volume $\left(\mathrm{dm}^{3}\right)$ was calculated from suspension length, average fore and rear widths, and average fore and rear depths.

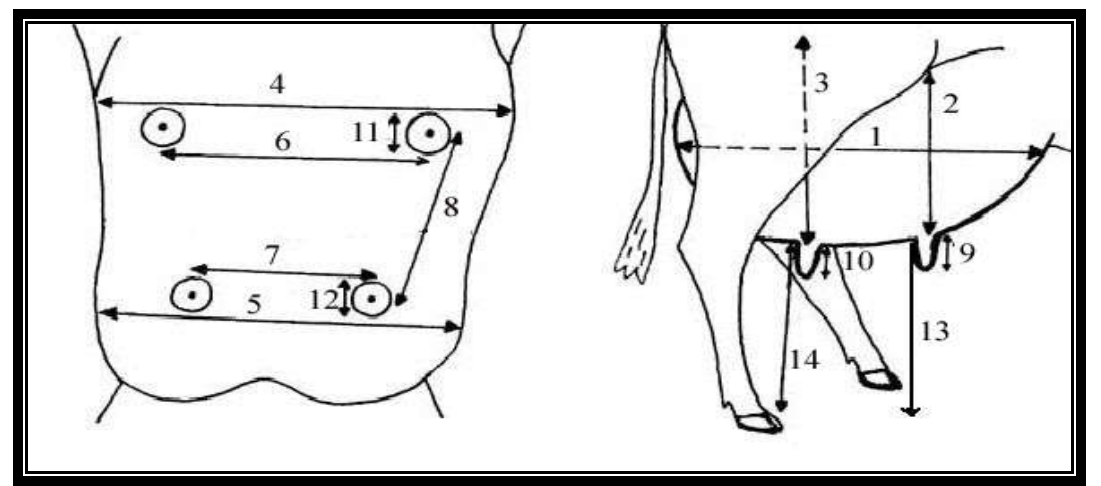

Figure 1. Udder measurements: 1- udder periphery, 2- udder fore depth, 3udder rear depth, 4- fore width, 5- rear width, 6- distance between fore teats, 7distance between rear teats, 8- distance between side teats, 9- length of fore teat, 10- length of rear teat, 11- diameter of fore teat, 12- diameter of rear teat, 13distance of fore quarters from the floor, 14- distance of rear quarters from the floor.

\section{Quantification of nucleic acids:}

Mammary cell number and its secretory activity were determined by using multiple biopsies to evaluate changes in DNA and RNA content, respectively. DNA and RNA changes in mammary tissue during the end of lactation and dry periods were quantitatively evaluated.

Mammary tissue biopsies were taken once from each cow for determinations of DNA and RNA from rear quarters by surgical method during the experimental period. Totals samples were taken as following, 4 samples were taken 7 days at $4 \mathrm{~h}$ after the milking before drying off, 4 samples were taken 14 days after drying off (the beginning of dry period), 2 samples were taken 35 days after drying off and also about 35 days before parturition (mid-dry period), and 4 samples were taken about 14 days before parturition (at the last of dry period).

DNA and RNA were isolated from tissue using E. Z. N. A. kits (Cat No. D349501 and R6634-01 for DNA and RNA, respectively) by Omega Bio-tek, Inc, USA). Determinations of DNA and RNA content, as indication for cell numbers and metabolic activity, were detected using spectrophotometer (Eppendorph BioPhotometer) according to enclosed pamphlet.

\section{Statistical analysis:}

Data were statistically analyzed using the general linear model procedure (SAS, 1999). The data was analyzed using the following model:

$$
\mathbf{Y}_{\mathrm{ijk}}=\boldsymbol{\mu}+\mathbf{P}_{\mathrm{i}}+\mathbf{L}_{\mathbf{j}}+\mathbf{P L}_{\mathrm{ij}}+\mathbf{e}_{\mathrm{ijk}}
$$


Where: $\mathbf{Y}_{\mathbf{i j k}}=$ an observation

$\boldsymbol{\mu}=$ overall means

$\mathbf{P}_{\mathbf{i}}=$ phase effect $(\mathrm{i}=1,2, \ldots ., 8)$

$\mathbf{L}_{\mathbf{j}}=$ length of dry period effect $(\mathrm{j}=1,2)$

$\mathbf{P L}_{\mathbf{i j}}=$ phase $\mathrm{x}$ length of dry period interaction effect

$\mathbf{e}_{\mathbf{i j k}}=$ random error

Significance among the means were checked using Duncan's Multiple Range Test (Duncan, 1955). Probability values $\leq 5 \%$ were considered significant.

\section{RESULTS AND DISCUSSION}

\section{Body weight:}

Data in Table (1) show that in general, the increased averages were 55.63 and $32.60 \mathrm{~kg}$ in body weight of cows during the long dry period (72.38 days) and the short dry period (33.25 days), respectively. This increase estimates with 10.62 and $6.01 \%$ from body weight at end of lactation, this body weight gain attributable to fetal growth during that period.

Cows having short dry period length lost $48.91 \mathrm{~kg}(8.50 \%)$ from their body weight at calving, while cows having long dry period length lost $41.41 \mathrm{~kg}(7.14 \%)$ from their body weight i.e. the cows having short dry period lost more from their body weight than cows having long dry period length. During postpartum cows body weight decreased insignificantly and it may be due to the beginning of milk production. Whereas cows having long dry period length lost $26.00 \mathrm{~kg}(4.83 \%)$ during the $1^{\text {st }}$ week, while cows having short dry period length lost $23.50 \mathrm{~kg}(4.47 \%)$.

These obtained result, agree with those obtained by Grum et al. (1996) who found that, the body weight gain attributable to fetal growth during the dry period was estimated to be 35 to $40 \mathrm{~kg}$. Also, Dann et al. (1999) and Park et al. (2002) found that, cows gained averages from 16.8 to $36.0 \mathrm{~kg}$ of body weight during the last 28 days of gestation. While, Gulay et al. (2003a) found that, cows lost averages from 13.1 to $15.7 \%$ of their body weight from week 1 through week 8 postpartum. Also, Gulay et al. (2003b) noticed that, at 2 week after calving, cows lost an additional $8.5 \%$ of their body weight, compared with the week of calving. During the following 2-week period, extent of body weight loss was less.

\section{Mammary gland features:}

\subsection{Distance of fore and rear quarters from the floor:}

Means of distance of fore and rear quarters from the floor $(\mathrm{cm})$ increased during the active and steady state involution (Table 1), until they reach the highest values during the steady state involution for cows having long dry period. Then, distance of fore quarters from the floor decreased significantly $(\mathrm{P}<0.05)$ during the redevelopment and colostrogenesis phase, but it was insignificant $(\mathrm{P}<0.05)$ for rear quarters. During postpartum period, distance of fore and rear quarters from the floor decreased gradually until they reach the lowest values. Generally, means of distance of fore and rear quarters from the floor were greater for cows having short dry period compared with those having long dry period during all study stages. These differences were significant $(\mathrm{P}<0.05)$ only at both the end of lactation and active involution periods (Table 1). 
Table 1. Changes in cow body weight and mammary gland dimensions in Friesian cows before, during dry and postpartum periods for cows having long and short dry period lengths

\begin{tabular}{|c|c|c|c|c|c|c|c|c|c|}
\hline \multirow{2}{*}{ Trait } & \multirow{2}{*}{$\begin{array}{l}\text { Dry } \\
\text { Period } \\
\text { Length }\end{array}$} & \multirow{2}{*}{ EL } & \multicolumn{3}{|c|}{ Dry Period (phase) } & \multicolumn{4}{|c|}{ Postpartum (day) } \\
\hline & & & $\mathbf{A I}$ & SSI & $\mathbf{R C}$ & $1^{\text {st }}$ & $2^{\text {nd }}$ & $3^{\text {rd }}$ & $7^{\text {th }}$ \\
\hline \multirow{4}{*}{$\begin{array}{l}\text { Body } \\
\text { weight } \\
\text { (kg) }\end{array}$} & \multirow{2}{*}{ Long } & $524.03 \pm$ & $536.09 \pm$ & $548.26 \pm$ & $579.66 \pm$ & $538.25 \pm$ & $529.88 \pm$ & $522.38 \pm$ & $512.25 \pm$ \\
\hline & & $40.71^{\mathrm{bA}}$ & $40.74^{\mathrm{bA}}$ & $32.62^{\mathrm{ab}}$ & $44.02^{\mathrm{aA}}$ & $41.89^{\mathrm{bA}}$ & $42.36^{\mathrm{bA}}$ & $42.74^{\mathrm{bA}}$ & $42.55^{\mathrm{bA}}$ \\
\hline & \multirow{2}{*}{ Short } & $542.56 \pm$ & $555.30 \pm$ & & $575.16 \pm$ & $526.25 \pm$ & $518.00 \pm$ & $511.25 \pm$ & $502.75 \pm$ \\
\hline & & $35.15^{\mathrm{bcA}}$ & $36.45^{\mathrm{abA}}$ & --- & $32.96^{\mathrm{aA}}$ & $28.76^{\mathrm{bcdA}}$ & $29.45^{\mathrm{cdA}}$ & $29.75^{\mathrm{dA}}$ & $29.23^{\mathrm{dA}}$ \\
\hline \multirow{4}{*}{ DFF } & \multirow{2}{*}{ Long } & $61.11 \pm$ & $63.69 \pm$ & $71.21 \pm$ & $61.05 \pm$ & $55.56 \pm$ & $54.50 \pm$ & $53.31 \pm$ & $52.13 \pm$ \\
\hline & & $7.33^{\mathrm{bcB}}$ & $7.79^{\mathrm{bB}}$ & $7.58^{\mathrm{a}}$ & $8.14^{\mathrm{bcA}}$ & $8.67^{\mathrm{cdA}}$ & $7.58^{\mathrm{cdA}}$ & $7.64^{\mathrm{dA}}$ & $6.06^{\mathrm{dA}}$ \\
\hline & \multirow{2}{*}{ Short } & $65.52 \pm$ & $69.80 \pm$ & & $63.32 \pm$ & $58.38 \pm$ & $57.75 \pm$ & $57.88 \pm$ & $56.38 \pm$ \\
\hline & & $8.74^{\mathrm{abA}}$ & $9.62^{\mathrm{aA}}$ & --- & $10.67^{\mathrm{abcA}}$ & $9.12^{\mathrm{bcA}}$ & $8.38^{\mathrm{bcA}}$ & $6.79^{\mathrm{bcA}}$ & $6.59^{\mathrm{cA}}$ \\
\hline \multirow{4}{*}{ DRF } & \multirow{2}{*}{ Long } & $58.78 \pm$ & $61.08 \pm$ & $68.63 \pm$ & $58.95 \pm$ & $53.31 \pm$ & $52.25 \pm$ & $51.56 \pm$ & $49.50 \pm$ \\
\hline & & $7.37^{\mathrm{bcB}}$ & $8.26^{\mathrm{bB}}$ & $8.13^{\mathrm{a}}$ & $8.39^{\mathrm{bcA}}$ & $8.60^{\mathrm{cdA}}$ & $7.34^{\mathrm{cdA}}$ & $8.11^{\mathrm{dA}}$ & $5.98^{\mathrm{dA}}$ \\
\hline & \multirow{2}{*}{ Short } & $63.55 \pm$ & $67.58 \pm$ & & $61.16 \pm$ & $55.81 \pm$ & $55.44 \pm$ & $56.06 \pm$ & $54.06 \pm$ \\
\hline & & $8.39^{\mathrm{abA}}$ & $9.62^{\mathrm{aA}}$ & --- & $10.83^{\mathrm{abcA}}$ & $9.65^{\mathrm{bcA}}$ & $8.38^{\mathrm{bcA}}$ & $7.06^{\mathrm{bcA}}$ & $6.77^{\mathrm{cA}}$ \\
\hline \multirow{4}{*}{ UFD } & \multirow{2}{*}{ Long } & $26.44 \pm$ & $24.34 \pm$ & $19.26 \pm$ & $24.75 \pm$ & $30.63 \pm$ & $28.75 \pm$ & $29.13 \pm$ & $30.25 \pm$ \\
\hline & & $1.56^{\mathrm{bA}}$ & $2.99^{\mathrm{bA}}$ & $1.24^{\mathrm{c}}$ & $3.84^{\mathrm{bA}}$ & $2.26^{\mathrm{aA}}$ & $2.25^{\mathrm{aA}}$ & $1.55^{\mathrm{aA}}$ & $2.25^{\mathrm{aA}}$ \\
\hline & \multirow{2}{*}{ Short } & $26.09 \pm$ & $25.20 \pm$ & & $25.05 \pm$ & $28.88 \pm$ & $28.25 \pm$ & $27.50 \pm$ & $30.50 \pm$ \\
\hline & & $2.48^{\text {cdeA }}$ & $3.09^{\mathrm{deA}}$ & --- & $3.47^{\mathrm{eA}}$ & $1.46^{\mathrm{abA}}$ & $2.31^{\mathrm{abcA}}$ & $1.60^{\mathrm{bcdA}}$ & $1.51^{\mathrm{aA}}$ \\
\hline \multirow{4}{*}{ URD } & \multirow{2}{*}{ Long } & $24.13 \pm$ & $21.97 \pm$ & $17.21 \pm$ & $22.31 \pm$ & $28.38 \pm$ & $26.13 \pm$ & $27.25 \pm$ & $28.25 \pm$ \\
\hline & & $1.50^{\mathrm{cdA}}$ & $3.03^{\mathrm{dA}}$ & $1.44^{\mathrm{e}}$ & $3.68^{\mathrm{dA}}$ & $2.45^{\mathrm{aA}}$ & $1.96^{\mathrm{bcA}}$ & $1.39^{\mathrm{abA}}$ & $2.25^{\mathrm{abA}}$ \\
\hline & \multirow{2}{*}{ Short } & $23.41 \pm$ & $23.10 \pm$ & & $22.63 \pm$ & $26.50 \pm$ & $26.13 \pm$ & $25.38 \pm$ & $27.63 \pm$ \\
\hline & & $2.14^{\mathrm{bcA}}$ & $3.08^{\mathrm{bcA}}$ & --- & $3.70^{\mathrm{cA}}$ & $1.93^{\mathrm{aA}}$ & $2.10^{\mathrm{aA}}$ & $1.41^{\mathrm{abB}}$ & $1.41^{\mathrm{aA}}$ \\
\hline \multirow{4}{*}{ UFW } & \multirow{2}{*}{ Long } & $26.56 \pm$ & $23.69 \pm$ & $18.74 \pm$ & $27.38 \pm$ & $35.88 \pm$ & $34.75 \pm$ & $33.00 \pm$ & $31.75 \pm$ \\
\hline & & $2.92^{\mathrm{dA}}$ & $3.46^{\mathrm{eA}}$ & $1.19^{\mathrm{f}}$ & $5.17^{\mathrm{dA}}$ & $1.81^{\mathrm{aA}}$ & $1.83^{\mathrm{abA}}$ & $2.33^{\mathrm{bcA}}$ & $1.39^{\mathrm{cA}}$ \\
\hline & \multirow{2}{*}{ Short } & $25.34 \pm$ & $23.40 \pm$ & & $28.58 \pm$ & $32.50 \pm$ & $31.13 \pm$ & $30.38 \pm$ & $30.13 \pm$ \\
\hline & & $3.15^{\mathrm{cA}}$ & $2.87^{\mathrm{cA}}$ & --- & $3.92^{\mathrm{bA}}$ & $1.41^{\mathrm{aB}}$ & $1.46^{\mathrm{abB}}$ & $2.45^{\mathrm{abB}}$ & $2.23^{\mathrm{abA}}$ \\
\hline & & $21.41 \pm$ & $18.81 \pm$ & $13.53 \pm$ & $20.91 \pm$ & $27.63 \pm$ & $26.13 \pm$ & $24.75 \pm$ & $23.25 \pm$ \\
\hline URW & Long & $3.33^{\mathrm{deA}}$ & $2.88^{\mathrm{eA}}$ & $1.22^{\mathrm{f}}$ & $4.61^{\mathrm{deA}}$ & $2.07^{\mathrm{aA}}$ & $2.42^{\mathrm{abA}}$ & $2.31^{\mathrm{bcA}}$ & $2.12^{\operatorname{cd} A}$ \\
\hline URW & & $19.00 \pm$ & $17.85 \pm$ & & $21.74 \pm$ & $24.25 \pm$ & $22.50 \pm$ & $21.50 \pm$ & $21.25 \pm$ \\
\hline & Short & $2.78^{\mathrm{bcB}}$ & $2.52^{\mathrm{cA}}$ & --- & $4.29^{\mathrm{abA}}$ & $3.69^{\mathrm{aB}}$ & $3.42^{\mathrm{aB}}$ & $2.98^{\mathrm{abB}}$ & $2.82^{\mathrm{abA}}$ \\
\hline & I ong & $111.78 \pm$ & $98.00 \pm$ & $84.53 \pm$ & $114.94 \pm$ & $146.88 \pm$ & $142.25 \pm$ & $134.75 \pm$ & $129.88 \pm$ \\
\hline UP & Long & $11.99^{\mathrm{dA}}$ & $12.94^{\mathrm{eA}}$ & $4.65^{\mathrm{f}}$ & $22.16^{\mathrm{dA}}$ & $9.93^{\mathrm{aA}}$ & $8.66^{\mathrm{abA}}$ & $9.13^{\mathrm{bcA}}$ & $5.54^{\mathrm{cA}}$ \\
\hline UP & & $105.00 \pm$ & $96.15 \pm$ & & $117.58 \pm$ & $133.50 \pm$ & $126.13 \pm$ & $121.75 \pm$ & $121.13 \pm$ \\
\hline & Short & $11.72^{\mathrm{cB}}$ & $11.24^{\mathrm{cA}}$ & --- & $16.42^{\mathrm{bA}}$ & $6.70^{\mathrm{aB}}$ & $6.42^{\mathrm{abB}}$ & $7.48^{\mathrm{bB}}$ & $7.16^{\mathrm{bB}}$ \\
\hline & Iong & $19.68 \pm$ & $14.27 \pm$ & $7.68 \pm$ & $20.58 \pm$ & $39.20 \pm$ & $34.18 \pm$ & $31.46 \pm$ & $30.15 \pm$ \\
\hline & Long & $4.86^{\mathrm{cA}}$ & $5.56^{\mathrm{dA}}$ & $0.81^{\mathrm{e}}$ & $11.01^{\mathrm{cA}}$ & $6.22^{\mathrm{aA}}$ & $5.79^{\mathrm{abA}}$ & $4.48^{\mathrm{bA}}$ & $3.47^{\mathrm{bA}}$ \\
\hline UV & & $17.03 \pm$ & $14.07 \pm$ & & $20.77 \pm$ & $30.11 \pm$ & $26.36 \pm$ & $23.97 \pm$ & $26.06 \pm$ \\
\hline & Short & $4.82^{\mathrm{deB}}$ & $4.62^{\mathrm{eA}}$ & --- & $7.51^{\mathrm{cdA}}$ & $2.65^{\mathrm{aB}}$ & $2.54^{\mathrm{abB}}$ & $2.89^{\mathrm{bcB}}$ & $3.12^{\mathrm{abB}}$ \\
\hline $\mathrm{EL}=\mathrm{En}$ & of Lactat & & & $\begin{array}{l}\text { DFF = Dista } \\
\text { he floor }\end{array}$ & ce of fore $\mathrm{q}$ & uarters from & UFV & $=$ Udder $\mathrm{f}$ & re width \\
\hline $\mathrm{AI}=\mathrm{Ac}$ & ive Involu & ion & & $\begin{array}{l}\mathrm{DFF}=\text { Dista } \\
\text { he floor }\end{array}$ & hce of rear q & uarters from & URV & $=$ Udder 1 & ar width \\
\hline $\mathrm{SSI}=\mathrm{St}$ & ady State & nvolution & & $\mathrm{JFD}=\mathrm{Udde}$ & fore depth & & $\mathrm{UP}=$ & Udder per & phery \\
\hline $\begin{array}{l}\mathrm{RC}=\mathrm{Re} \\
\text { Colostro }\end{array}$ & $\begin{array}{l}\text { developme } \\
\text { genesis }\end{array}$ & nt and & & $\mathrm{JRD}=\mathrm{Udd}$ & $r$ rear depth & & UV & Udder vo & ame \\
\hline
\end{tabular}

Table (1) shows that, means of udder fore and rear depths $(\mathrm{cm})$ were decreased gradually during the active and steady state involution phases for cows having long and short dry periods, and they reached its lowest values $(19.26$ and $17.21 \mathrm{~cm})$ during the steady state involution for cows having long dry period, respectively. While 
during the redevelopment and colostrogenesis phase they increased significantly $(\mathrm{P}<0.05)$ for cows having long dry period $(24.75$ and $22.31 \mathrm{~cm}$, respectively), but for cows having short dry period it decreased insignificantly $(\mathrm{P}<0.05)$ and attained 25.05 and $22.63 \mathrm{~cm}$, respectively. At the $1^{\text {st }}$ day postpartum, they increased significantly $(\mathrm{P}<0.05)$ for both cows having long and short dry periods. Differences between means of udder fore and rear depths for cows having long and short dry period lengths during all study stages were not significant (Table 1).

\subsection{Udder fore and rear widths:}

Table (1) showed that, means of udder fore and rear widths $(\mathrm{cm})$ decreased during active and steady state involution phases for cows having long and short dry periods, reached its lowest values. While it increased significantly $(\mathrm{P}<0.05)$ at the redevelopment and colostrogenesis phase and the $1^{\text {st }}$ day postpartum for cows having long and short dry periods. After that, they began to steady decrease to the end of the $7^{\text {th }}$ day postpartum. Means of udder fore and rear widths were not different significantly $(\mathrm{P}<0.05)$ between cows having long and short dry period lengths during the end of lactation, dry periods and at the $7^{\text {th }}$ day postpartum, but during colostrum period $\left(1^{\text {st }}\right.$ to $3^{\text {rd }}$ day postpartum) they increased significantly $(\mathrm{P}<0.05)$ for cows having long dry period length (Table 1$)$.

\subsection{Udder periphery and udder volume:}

Table (1) shows mean of udder periphery $(\mathrm{cm})$ and volume $\left(\mathrm{dm}^{3}\right)$. Means of udder periphery decreased during active and steady state involution phases for cows having long and short dry periods, and they reach the lowest values $(84.53 \mathrm{~cm})$ during the steady state involution for cows having long dry period. While during the redevelopment and colostrogenesis phase and at the $1^{\text {st }}$ day postpartum it increased significantly $(\mathrm{P}<0.05)$ for cows having long and short dry periods. After that, they began to decrease gradually. During the dry period, means of udder periphery were not differ significantly between cows having long and short dry period lengths. But they were significant $(\mathrm{P}<0.05)$ for cows having long dry period length during end of lactation and postpartum periods.

Means of udder volume decreased significantly $(\mathrm{P}<0.05)$ during the active involution $(27.49 \%)$ and the steady state involution $(46.18 \%)$ phases for cows having long dry period, while it decreased insignificantly during active involution $(17.38 \%)$ for cows having short dry period (Table 1). During redevelopment and colostrogenesis phase and at the $1^{\text {st }}$ day postpartum it increased significantly $(\mathrm{P}<0.05)$ for cows having long and short dry periods. This increase represents 167.97 and $90.48 \%$ for cows having long dry period, respectively. While it constitutes $47.62 \%$ and $44.97 \%$ for cows having short dry period, respectively. Then, udder volume decreased insignificantly. Means of udder volume was significant $(\mathrm{P}<0.05)$ for cows having long dry period length during the end of lactation and postpartum periods. But they were not significant between cows having long and short dry period lengths during dry period (Table 1).

\subsection{Fore and rear teats lengths:}

Table (2) shows that, means of fore and rear teats lengths $(\mathrm{cm})$ decreased during the active and steady state involution phases for cows having long and short dry 
periods, and they reached their lowest values during steady state involution for cows having long dry period. While during the redevelopment and colostrogenesis phase and at the $1^{\text {st }}$ day postpartum they increased significantly $(\mathrm{P}<0.05)$ for cows having long dry period, but not significantly for cows having short dry period. However differences between them during the residual postpartum days were not significant.

\subsection{Diameter of fore and rear teats:}

Table (2) shows that, means of diameter of fore and rear teats $(\mathrm{cm})$ decreased during active and steady state involution phases for cows having long and short dry periods, reaching the lowest values during the steady state involution for cows having long dry period, respectively. While during the redevelopment and colostrogenesis phase and at the $1^{\text {st }}$ day postpartum they increased significantly $(\mathrm{P}<0.05)$ for cows having long dry period, but insignificantly for cows having short dry period and during the $1^{\text {st }}$ week postpartum. During the end of lactation they increased significantly $(\mathrm{P}<0.05)$ for cows having long dry period compared with those having short dry period. But not were significant between them during both dry and postpartum periods (Table 2).

\subsection{Distances between teats:}

Table (2) shows that, means of distance between fore teats, distance between rear teats and distance between side teats $(\mathrm{cm})$ decreased during the active and steady state involution phases for cows having long and short dry periods, and they reached the lowest values during steady state involution for cows having long dry period, respectively. While during redevelopment and colostrogenesis phase and at $1^{\text {st }}$ day postpartum they increased significantly $(\mathrm{P}<0.05)$ for cows having long and short dry periods. After that, no significant differences were observed during $1^{\text {st }}$ week postpartum. During $3^{\text {rd }}$ and $7^{\text {th }}$ day postpartum distance between fore teats increased significantly $(\mathrm{P}<0.05)$ for cows having long dry period compared with cows having short dry period. But not differences between cows having long and short dry period lengths during end of lactation, dry periods, at $1^{\text {st }}$ and $2^{\text {nd }}$ day postpartum. As well as between them during all study stages for means of distance between rear teats and distance between side teats.

Seykora and McDaniel (1986); El-Gaafarawy et al. (1996); and Kuczaj (2003) studied the evolution of the morphological traits of the udder and teat, and indicated that physical characteristics of the udder and teats were related to economically important traits in dairy cows. Shape and attachments of the udder and size, shape and placement of the teats are generally considered as important characters for dairy cows.

Also, El-Gaafarawy et al. (1996) found that, least-squares means of accumulated milk yield and udder dimensions (depth, diagonal and perimeter) increased with advance of parity. Teat dimensions (diameter and length) increased from first parity to third and later ones. While, Kuczaj (2003) noted that, udder volume in the $3^{\text {rd }}$ lactation in comparison with the $1^{\text {st }}$ lactation increased by $47.7 \%$ (Sawicki and Kijak, 1984), or by $54.27 \%$ (Pawlina et al., 2000), or even by 67.6\% (Litwińczuk, 1986). As a consequence of udder depth increase, the distance from the udder to the floor was reduced. 
Table 2. Changes in teat traits in cows before and during the dry period and postpartum period for Friesian cows having long and short dry period lengths

\begin{tabular}{|c|c|c|c|c|c|c|c|c|c|}
\hline \multirow[t]{2}{*}{ Trait } & \multirow{2}{*}{$\begin{array}{l}\text { Dry } \\
\text { Period } \\
\text { Length }\end{array}$} & \multirow{2}{*}{$\mathbf{E L}$} & \multicolumn{3}{|c|}{ Dry Period (phase) } & \multicolumn{4}{|c|}{ Postpartum (day) } \\
\hline & & & AI & SSI & $\mathbf{R C}$ & $1^{\text {st }}$ & $2^{\text {nd }}$ & $3^{\text {rd }}$ & $7^{\text {th }}$ \\
\hline \multirow{2}{*}{ LFT } & Long & $\begin{array}{l}5.39 \pm \\
0.76^{\text {bcA }}\end{array}$ & $\begin{array}{l}5.05 \pm \\
0.80^{\mathrm{cA}}\end{array}$ & $\begin{array}{c}4.42 \pm \\
0.38^{\mathrm{d}}\end{array}$ & $\begin{array}{l}5.70 \pm \\
0.82^{\text {bA }}\end{array}$ & $\begin{array}{l}6.44 \pm \\
0.56^{\mathrm{aA}}\end{array}$ & $\begin{array}{l}6.50 \pm \\
0.38^{\mathrm{aA}}\end{array}$ & $\begin{array}{l}6.38 \pm \\
0.44^{\mathrm{aA}}\end{array}$ & $\begin{array}{l}6.44 \pm \\
0.50^{\mathrm{aA}}\end{array}$ \\
\hline & Short & $\begin{array}{l}5.33 \pm \\
0.69^{\text {bca }}\end{array}$ & $\begin{array}{l}5.15 \pm \\
0.67^{\mathrm{cA}}\end{array}$ & --- & $\begin{array}{c}5.61 \pm \\
0.74^{\text {abcA }}\end{array}$ & $\begin{array}{l}6.13 \pm \\
0.69^{\mathrm{aA}}\end{array}$ & $\begin{array}{l}6.00 \pm \\
0.46^{\mathrm{aB}}\end{array}$ & $\begin{array}{l}5.88 \pm \\
0.64^{\mathrm{abA}}\end{array}$ & $\begin{array}{l}5.94 \pm \\
0.50^{\mathrm{abA}}\end{array}$ \\
\hline \multirow{2}{*}{ LRT } & Long & $\begin{array}{l}4.91 \pm \\
0.77^{\mathrm{bA}}\end{array}$ & $\begin{array}{l}4.95 \pm \\
0.68^{\mathrm{bA}}\end{array}$ & $\begin{array}{c}4.18 \pm \\
0.34^{\mathrm{c}}\end{array}$ & $\begin{array}{l}5.25 \pm \\
0.81^{\mathrm{bA}}\end{array}$ & $\begin{array}{l}6.13 \pm \\
0.44^{\mathrm{aA}}\end{array}$ & $\begin{array}{l}6.13 \pm \\
0.44^{\mathrm{aA}}\end{array}$ & $\begin{array}{l}5.94 \pm \\
0.32^{\mathrm{aA}}\end{array}$ & $\begin{array}{l}6.00 \pm \\
0.53^{\mathrm{aA}}\end{array}$ \\
\hline & Short & $\begin{array}{l}4.83 \pm \\
0.59^{\mathrm{bA}}\end{array}$ & $\begin{array}{l}4.83 \pm \\
0.73^{\mathrm{bA}}\end{array}$ & --- & $\begin{array}{c}5.18 \pm \\
0.77^{\mathrm{abA}}\end{array}$ & $\begin{array}{l}5.75 \pm \\
0.53^{\mathrm{aA}}\end{array}$ & $\begin{array}{l}5.50 \pm \\
0.38^{\mathrm{aB}}\end{array}$ & $\begin{array}{l}5.25 \pm \\
0.60^{\mathrm{abB}}\end{array}$ & $\begin{array}{l}5.44 \pm \\
0.62^{\mathrm{aA}}\end{array}$ \\
\hline \multirow{2}{*}{ DFT } & Long & $\begin{array}{l}2.07 \pm \\
0.19^{\mathrm{abA}}\end{array}$ & $\begin{array}{l}1.96 \pm \\
0.22^{\text {bca }}\end{array}$ & $\begin{array}{l}1.75 \pm \\
0.14^{\mathrm{d}}\end{array}$ & $\begin{array}{l}1.87 \pm \\
0.22^{\mathrm{dcA}}\end{array}$ & $\begin{array}{l}2.19 \pm \\
0.21^{\mathrm{aA}}\end{array}$ & $\begin{array}{l}2.11 \pm \\
0.17^{\mathrm{abA}}\end{array}$ & $\begin{array}{l}2.15 \pm \\
0.17^{\mathrm{aA}}\end{array}$ & $\begin{array}{l}2.15 \pm \\
0.17^{\mathrm{aA}}\end{array}$ \\
\hline & Short & $\begin{array}{l}1.96 \pm \\
0.19^{\mathrm{bB}}\end{array}$ & $\begin{array}{l}1.92 \pm \\
0.11^{\mathrm{bA}}\end{array}$ & --- & $\begin{array}{l}1.96 \pm \\
0.14^{\mathrm{bA}}\end{array}$ & $\begin{array}{l}2.15 \pm \\
0.24^{\mathrm{aA}}\end{array}$ & $\begin{array}{l}2.07 \pm \\
0.23^{\mathrm{abA}}\end{array}$ & $\begin{array}{l}2.03 \pm \\
0.14^{\text {abA }}\end{array}$ & $\begin{array}{l}2.05 \pm \\
0.16^{\mathrm{abA}}\end{array}$ \\
\hline \multirow{2}{*}{ DRT } & Long & $\begin{array}{c}1.95 \pm \\
0.19^{\mathrm{abA}}\end{array}$ & $\begin{array}{c}1.83 \pm \\
0.22^{\mathrm{bcA}}\end{array}$ & $\begin{array}{l}1.62 \pm \\
0.15^{\mathrm{d}}\end{array}$ & $\begin{array}{c}1.74 \pm \\
0.24^{\mathrm{cdA}}\end{array}$ & $\begin{array}{l}2.07 \pm \\
0.21^{\mathrm{aA}}\end{array}$ & $\begin{array}{l}2.01 \pm \\
0.17^{\mathrm{aA}}\end{array}$ & $\begin{array}{c}1.99 \pm \\
0.17^{\mathrm{abA}}\end{array}$ & $\begin{array}{l}2.07 \pm \\
0.19^{\mathrm{aA}}\end{array}$ \\
\hline & Short & $\begin{array}{l}1.83 \pm \\
0.19^{\mathrm{abB}}\end{array}$ & $\begin{array}{l}1.79 \pm \\
0.11^{\mathrm{bA}}\end{array}$ & --- & $\begin{array}{c}1.83 \pm \\
0.15^{\mathrm{abA}}\end{array}$ & $\begin{array}{l}1.99 \pm \\
0.24^{\mathrm{aA}}\end{array}$ & $\begin{array}{c}1.93 \pm \\
0.22^{\mathrm{abA}}\end{array}$ & $\begin{array}{l}1.91 \pm \\
0.12^{\mathrm{abA}}\end{array}$ & $\begin{array}{c}1.91 \pm \\
0.15^{\text {abA }}\end{array}$ \\
\hline \multirow{2}{*}{ DF } & Long & $\begin{array}{l}13.14 \pm \\
2.05^{\mathrm{cbA}}\end{array}$ & $\begin{array}{c}11.67 \pm \\
2.13^{\mathrm{cA}}\end{array}$ & $\begin{array}{l}9.58 \pm \\
1.12^{\mathrm{d}}\end{array}$ & $\begin{array}{c}15.05 \pm \\
3.62^{\mathrm{bA}}\end{array}$ & $\begin{array}{c}19.38 \pm \\
2.56^{\mathrm{aA}}\end{array}$ & $\begin{array}{c}17.88 \pm \\
2.31^{\mathrm{aA}}\end{array}$ & $\begin{array}{c}17.69 \pm \\
1.94^{\mathrm{aA}}\end{array}$ & $\begin{array}{c}17.75 \pm \\
2.00^{\mathrm{aA}}\end{array}$ \\
\hline & Short & $\begin{array}{l}12.97 \pm \\
3.18^{\text {bcA }}\end{array}$ & $\begin{array}{c}11.25 \pm \\
3.50^{\mathrm{cA}}\end{array}$ & --- & $\begin{array}{l}14.37 \pm \\
3.30^{\mathrm{abA}}\end{array}$ & $\begin{array}{c}16.63 \pm \\
3.25^{\mathrm{aA}}\end{array}$ & $\begin{array}{l}15.63 \pm \\
2.13^{\mathrm{abA}}\end{array}$ & $\begin{array}{l}14.94 \pm \\
2.03^{\mathrm{abB}}\end{array}$ & $\begin{array}{l}15.06 \pm \\
2.08^{\mathrm{abB}}\end{array}$ \\
\hline \multirow{2}{*}{ DR } & Long & $\begin{array}{l}8.33 \pm \\
1.32^{\mathrm{cA}}\end{array}$ & $\begin{array}{l}6.89 \pm \\
1.44^{\mathrm{cdA}}\end{array}$ & $\begin{array}{c}5.50 \pm \\
0.60^{\mathrm{d}}\end{array}$ & $\begin{array}{c}10.55 \pm \\
3.20^{\mathrm{bA}}\end{array}$ & $\begin{array}{c}12.38 \pm \\
1.09^{\mathrm{aA}}\end{array}$ & $\begin{array}{l}11.75 \pm \\
1.46^{\mathrm{abA}}\end{array}$ & $\begin{array}{l}11.81 \pm \\
2.31^{\mathrm{abA}}\end{array}$ & $\begin{array}{l}12.06 \pm \\
2.19^{\mathrm{abA}}\end{array}$ \\
\hline & Short & $\begin{array}{l}7.70 \pm \\
1.66^{\mathrm{cA}}\end{array}$ & $\begin{array}{l}6.48 \pm \\
1.51^{\mathrm{cA}}\end{array}$ & --- & $\begin{array}{l}9.82 \pm \\
2.19^{\mathrm{bA}}\end{array}$ & $\begin{array}{c}11.81 \pm \\
1.10^{\mathrm{aA}}\end{array}$ & $\begin{array}{c}11.50 \pm \\
1.00^{\mathrm{aA}}\end{array}$ & $\begin{array}{c}11.69 \pm \\
0.75^{\mathrm{aA}}\end{array}$ & $\begin{array}{c}11.75 \pm \\
0.65^{\mathrm{aA}}\end{array}$ \\
\hline \multirow{2}{*}{ DS } & Long & $\begin{array}{l}8.84 \pm \\
1.63^{\operatorname{cdA}}\end{array}$ & $\begin{array}{l}7.27 \pm \\
1.69^{\mathrm{dA}}\end{array}$ & $\begin{array}{c}5.45 \pm \\
0.69^{\mathrm{e}}\end{array}$ & $\begin{array}{l}9.50 \pm \\
2.85^{\mathrm{cA}}\end{array}$ & $\begin{array}{c}13.50 \pm \\
3.02^{\mathrm{aA}}\end{array}$ & $\begin{array}{l}12.50 \pm \\
2.84^{\mathrm{abA}}\end{array}$ & $\begin{array}{l}11.94 \pm \\
2.56^{\mathrm{abA}}\end{array}$ & $\begin{array}{c}11.56 \pm \\
2.44^{\mathrm{bA}}\end{array}$ \\
\hline & Short & $\begin{array}{l}8.63 \pm \\
1.73^{\mathrm{cA}} \\
\end{array}$ & $\begin{array}{l}6.78 \pm \\
1.53^{\mathrm{dA}}\end{array}$ & --- & $\begin{array}{l}9.32 \pm \\
2.23^{\text {bcA }}\end{array}$ & $\begin{array}{c}12.13 \pm \\
2.36^{\mathrm{aA}} \\
\end{array}$ & $\begin{array}{c}11.38 \pm \\
2.08^{\mathrm{aA}} \\
\end{array}$ & $\begin{array}{c}11.06 \pm \\
1.88^{\mathrm{aA}} \\
\end{array}$ & $\begin{array}{l}10.75 \pm \\
1.73^{\text {abA }}\end{array}$ \\
\hline \multirow{3}{*}{\multicolumn{5}{|c|}{$\begin{array}{l}\text { EL = End of Lactation } \\
\text { AI = Active Involution } \\
\text { SSI = Steady State Involution }\end{array}$}} & $\mathrm{DFT}=\mathrm{D}$ & imeter of & re teat & & \\
\hline & & & & & $\mathrm{DRT}=\mathrm{L}$ & ameter of & ear teat & & \\
\hline & & & & & $\mathrm{DF}=\mathrm{Di}$ & ance betw & en fore tea & & \\
\hline \multicolumn{5}{|c|}{$\mathrm{RC}=$ Redevelopment and Colostrogenesis } & $\mathrm{DR}=\mathrm{Di}$ & tance betw & en rear tes & & \\
\hline \multicolumn{5}{|c|}{ LFT $=$ Length of fore teat } & $\mathrm{DS}=\operatorname{dis}$ & ance betwe & $\mathrm{n}$ side tea & & \\
\hline \multicolumn{10}{|c|}{ LRT $=$ Length of rear teat } \\
\hline
\end{tabular}

\section{Quantification of nucleic acids:}

During the end of lactation the average concentrations of DNA were 3.25 and $3.15 \mathrm{mg} / \mathrm{g}$ decreased to 3.00 and $2.86 \mathrm{mg} / \mathrm{g}$ during the active involution phase for cows given long and short dry period lengths, respectively (Table 3 ). This would suggest that milk stasis during the active involution has been demonstrated to stimulate DNA laddering in mammary tissue. These observations indicate that mammary epithelial cells are indeed lost during involution in the mammary gland. However, this process of cell loss does not seem to be as dramatic. Concentrations of DNA decreased by 7.69 and $9.21 \%$ from the end of lactation to active involution for cows having long and short dry periods, respectively. This indicate that mammary 
epithelial cells had a greater loss in mammary tissue for cows having short dry period during this period than cows having long dry period.

While during the steady state involution phase for cows having long dry period length concentration of DNA decreased to $2.87 \mathrm{mg} / \mathrm{g}$ (by $11.69 \%$ from the end of lactation). These indicate that mammary epithelial cells loss during active involution and steady state involution phases were greater in mammary tissue for cows having long dry period.

Table 3. Means \pm SE of DNA, RNA concentrations (mg/g), and RNA: DNA ratio of the mammary gland tissue before and during dry periods for Friesian cows having long and short dry period lengths

\begin{tabular}{|c|c|c|c|c|c|}
\hline \multirow{2}{*}{ Component } & \multirow{2}{*}{$\begin{array}{l}\text { Dry Period } \\
\text { Length }\end{array}$} & \multirow{2}{*}{$\mathbf{E L}$} & \multicolumn{3}{|c|}{ Dry Period (phase) } \\
\hline & & & $\mathbf{A I}$ & SSI & $\mathbf{R C}$ \\
\hline \multirow{2}{*}{$\begin{array}{l}\text { DNA } \\
(\mathrm{mg} / \mathrm{g})\end{array}$} & Long & $\begin{array}{l}3.25 \pm \\
0.31^{\mathrm{abA}}\end{array}$ & $\begin{array}{l}3.00 \pm \\
0.21^{\mathrm{abA}}\end{array}$ & $\begin{array}{l}2.87 \pm \\
0.27^{\mathrm{b}}\end{array}$ & $\begin{array}{l}3.91 \pm \\
0.46^{\mathrm{aA}}\end{array}$ \\
\hline & Short & $\begin{array}{l}3.15 \pm \\
0.25^{\mathrm{aA}}\end{array}$ & $\begin{array}{l}2.86 \pm \\
0.16^{\mathrm{aA}}\end{array}$ & --- & $\begin{array}{l}3.62 \pm \\
0.57^{\mathrm{aA}}\end{array}$ \\
\hline \multirow{2}{*}{$\begin{array}{l}\text { RNA } \\
(\mathrm{mg} / \mathrm{g})\end{array}$} & Long & $\begin{array}{l}5.71 \pm \\
1.00^{\mathrm{aA}}\end{array}$ & $\begin{array}{l}4.22 \pm \\
0.57^{\text {abA }}\end{array}$ & $\begin{array}{l}3.01 \pm \\
0.55^{\mathrm{b}}\end{array}$ & $\begin{array}{l}6.01 \pm \\
0.44^{\mathrm{aA}}\end{array}$ \\
\hline & Short & $\begin{array}{l}5.91 \pm \\
0.42^{\mathrm{aA}}\end{array}$ & $\begin{array}{l}4.80 \pm \\
0.76^{\mathrm{aA}}\end{array}$ & --- & $\begin{array}{l}6.26 \pm \\
0.36^{\mathrm{aA}}\end{array}$ \\
\hline \multirow{2}{*}{ RNA: DNA } & Long & $\begin{array}{l}1.75 \pm \\
0.14^{\mathrm{aA}}\end{array}$ & $\begin{array}{l}1.41 \pm \\
0.09^{\mathrm{bA}}\end{array}$ & $\begin{array}{l}1.05 \pm \\
0.09^{\mathrm{c}}\end{array}$ & $\begin{array}{l}1.54 \pm \\
0.07^{\mathrm{abA}}\end{array}$ \\
\hline & Short & $\begin{array}{l}1.88 \pm \\
0.02^{\mathrm{aA}}\end{array}$ & $\begin{array}{l}1.68 \pm \\
0.18^{\mathrm{aA}}\end{array}$ & --- & $\begin{array}{l}1.74 \pm \\
0.17^{\mathrm{aA}}\end{array}$ \\
\hline $\begin{array}{l}\text { EL = End of } \mathrm{I} \\
\mathrm{SSI}=\text { Steady } \\
\mathrm{a}, \mathrm{b}, \mathrm{c}: \text { Means in }\end{array}$ & $\begin{array}{l}\text { tation } \\
\text { te Involution }\end{array}$ & & $\begin{array}{l}\mathrm{AI}=\text { Activ } \\
\mathrm{RC}=\text { Rede } \\
\mathrm{s} \text { are signifi }\end{array}$ & $\begin{array}{l}\text { lution } \\
\text { nent and } \\
\text { different }\end{array}$ & $\begin{array}{l}\text { rogenesis } \\
05) \text {. }\end{array}$ \\
\hline
\end{tabular}

The average concentrations of DNA during the redevelopment and colostrogenesis phase increased to 3.91 and $3.62 \mathrm{mg} / \mathrm{g}$ (by 20.31 and $14.92 \%$ from the end of lactation) for cows having long and short dry periods, respectively. This demonstrates that mammary epithelial cells increased during this phase and this increase was greater in mammary tissue for cows having long dry period (Table 3 ).

During the end of lactation the average concentrations of RNA were 5.71 and $5.91 \mathrm{mg} / \mathrm{g}$ decreased to 4.22 and $4.80 \mathrm{mg} / \mathrm{g}$ during the active involution for cows having long and short dry period lengths, respectively. Then decreased to $3.01 \mathrm{mg} / \mathrm{g}$ during the steady state involution for cows having long dry period length (Table 3 ). This demonstrates that secretory activity for mammary tissue decreased gradually during the active and steady state involution phases. Also, during the redevelopment and colostrogenesis phase concentrations of RNA were increased to 6.10 and 6.26 $\mathrm{mg} / \mathrm{g}$ for cows having long and short dry periods, respectively.

The ratio of RNA: DNA was greater for cows having short dry period throughout the experimental period. This show that, secretory activity for mammary epithelial cells was greater for cows having short dry period compared with cows having long dry period. 
These data suggest that the importance of the dry period might be to repair or replace damaged or senescent mammary epithelial cells prior to the next lactation.

Knight and Peaker (1984) assessed the mammary cell number and secretory activity during lactation of goats. This study demonstrated that increases in milk production during early lactation were firstly the result of an increase in mammary cell number followed by an increase in secretory activity per cell. After peak of lactation, decreased milk yield with advancing lactation was primarily the result of declining cell number. Also during late lactation, when goats were concomitantly pregnant, the secretory activity per cell also declined.

Capuco et al. (1997) studied the influence of the dry period on mammary growth during sixty days before expected parturition. They found that, the ratio of RNA: DNA tended to be greater for lactating cows than for dry cows. Also, they found that greater proportion of epithelial cells in dry glands than in lactating glands during the final week of gestation.

Capuco et al. (2001 and 2003) stated that, when cows are concomitantly lactating and pregnant, it is likely that a decline in secretory capacity per mammary cell accompanies advanced pregnancy, due to the conflicting metabolic demands of gestation and lactation. Hurley and Ford (2002) stated that, once the animal becomes pregnant, development of the mammary gland accelerates at an exponential rate. This means that mammary growth is greatest during the later stages of pregnancy. In cows, mammary DNA increases by $65 \%$ between 10 days prepartum and 10 days postpartum.

Hurley (2002) indicated that, removal of secretory epithelial cells by apoptosis is a normal physiological event in the ruminant mammary gland, even during lactation. In addition, milk stasis has been demonstrated to stimulate DNA laddering in cow mammary tissue. Also, he indicated that, cows given a 60 days dry period, an increase in mammary DNA synthesis begins occurring about 35 days prepartum. This might indicate that the early stages of redevelopment are beginning even 5 weeks prepartum.

Grummer and Rastani (2004) indicated that, Milk yield is a function of the number of secretory cells and the activity of those cells. Cell numbers reflect a balance between cell proliferation and apoptosis (programmed cell death). In nonpregnant cows, the decline in milk production as lactation progresses is due to changes in cell number rather than to changes in cell activity. If cows are pregnant, the decline in production may also be related to decrease secretory activity.

\section{ACKNOWLEDGMENT}

Deep thanks and sincere appreciation to Dr. Ahmed R. A. Elbeltagi, Researcher of Animal Breeding, Biotechnology Department, Animal Production Research Institute, for his excellent facilities and helping in isolation and determination of DNA and RNA. 


\section{REFERENCES}

Capuco, A. V., R. M. Akers, and J. J. Smith, 1997. Mammary growth in Holstein cows during the dry period: quantification of nucleic acids and histology. J. Dairy Sci., 80:477-487.

Capuco, A.V., D.L. Wood, R. Baldwin, K. Mcleod, and M.J. Paape, 2001. Mammary cell number, proliferation, and apoptosis during a bovine lactation: relation to milk production and effect of bST. J. Dairy Sci., 84: 2177-2187.

Capuco, A.V., S.E. Ellis, S.A. Hale, E. Long, R.A. Erdman, X. Zhao, and M.J. Paape. (2003). Lactation persistency: Insights from mammary cell proliferation studies. J. Anim. Sci., 81(Suppl. 3): 18-31.

Dann, H.M., G.A. Varga and D.E. Putnam, 1999. Improving energy supply to late gestation and early postpartum dairy cows. J. Dairy Sci., 82: 1765-1778.

Duncan, D.B., 1955. Multiple range and multiple F. test. Biometrics, 11: 1-24.

El-Gaafarawy, A.M., S.M. Zahed and E.A. Omar, 1996. Milking flow characteristics, udder and teat dimensions and their relationship with milk yield of Friesian cows raised in Egypt. Egyptian J. Anim. Prod., 33: Suppl. Issue: 1-18.

Grum, D.E., J.K. Drackley, R.S. Younker, D.W. LaCount, and J.J. Veenhuizen. (1996). Nutrition during the dry period and hepatic lipid metabolism of periparturient dairy cows. J. Dairy Sci., 79: 1850-1864.

Grummer, R.R. and R.R. Rastani, 2004. Why Reevaluate Dry Period Length? J. Dairy Sci., 87: (E. Suppl.): E77-E85.

Gulay, M.S., M.J. Hayen, K.C. Bachman, T. Belloso, M. Liboni, and H.H. Head, 2003a. Milk production and feed intake of Holstein cows given short (30-d) or normal (60-d) dry periods. J. Dairy Sci., 86: 2030-2038.

Gulay, M.S., M.J. Hayen, L.C. Teixeira, C.J. Wilcox, and H.H. Head, 2003b. Responses of Holstein cows to a low dose of somatotropin (bST) prepartum and postpartum. J. Dairy Sci., 86: 3195-3205.

Hurley, W.L., 2002. Lactation Biology: Mammary gland involution and dry period. Department of Animal Sciences, University of Illinois, Urbana-Champaign. http://www.classes. aces.uiuc. edu/An Sci308/involution.html Accessed July 26, 2003.

Hurley, W. L. and J. A. Ford Jr, 2002. Mammary gland: growth, development and involution. In: Encyclopedia of Dairy Sciences, H. Roginski, J. W. Fuquay and P. F. Fox,(eds) Academic Press, New York, USA, pp. 1689-1697.

Knight, C. H. and M. Peaker, 1984. Mammary development and regression during lactation in goats in relation to milk secretion. Q. J. Exp. Physiol., 69:331-338.

Kuczaj, M., 2003. Analysis of changes in udder size of high-yelding cows in subsequent lactations with regard to mastitis. Electronic J. Polish Agric. Univ., Anim. Husb., 6: 1. http://www. ejpau.media.pl/series/volume6/issuel/animal/art02.html Accessed December 20, 2004.

Litwińczuk, Z., 1986. Changes in external structure of udders and rapidity of milking in cows in relation to age and course of lactation. Med. Weter., 42: 176-180 (Cited by Kuczaj, 2003).

Park, A. F., J. E. Shirley, E. C. Titgemeyer, M. J. Meyer, M. J. VanBaale, and M. J. VandeHaar, 2002. Effect of protein level in prepartum diets on metabolism and performance of dairy cows. J. Dairy Sci., 85: 1815-1828. 
Pawlina, E.,W. Kruszyński, and M. Kuczaj, 2000. The changes in udder size of Red and White breed cows in first and third lactation. Med. Weter. 56: 672-674 (Cited by Kuczaj, 2003).

SAS (1999).User's Guide: Statistics, Version 8.0 Edition. SAS Inst., Inc., Cary, NC.

Sawicki, J. and Z. Kijak, 1984. Effect of successive lactations of cows kept in large cattle farms on udder structure. Zesz. Nauk. ART Olszt. Ser. Zootech. 26: 31-39 (Cited by Kuczaj, 2003).

Seykora, A.J., and B.T. McDaniel, 1986. Genetic statistics and relationships of teat and udder traits, somatic cell counts and milk production. J. Dairy Sci., 69: 23952407.

Zhang, W.C., J.C.M. Dekkers, G. Banos and E.B. Burnside, 1994. Adjustment factors and genetic evaluation for somatic cell score and relationship with other traits of Canadian Holstein. J. Dairy Sci., 77, 659-665. 
الغدة اللبنية في الأبقار خلال فترة الجفاف: r. التغيرات في وزن الجسم والخصائص

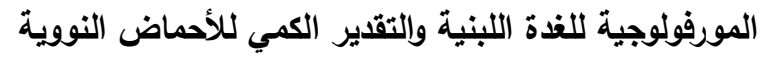

أحمد سليمان محمود سليمان' ،عبد الكريم ابراهيم محمد السيد' ، أحمد محمد الجعفروي" ، ممدوح علي السيد علي أحمب سلن

1 - قسم الإنتاج الحيوانس، كلية النزاعة، جامعة بنها، ب - معهج بحوث الإنتاج الحيوانس، مركز البحوث

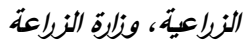

الهدف من هذه الدراسة هو معرفة التغرات التي تحدث في وزن الجسم ، الخصائص المورفولوجية للغدة

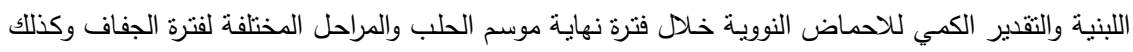

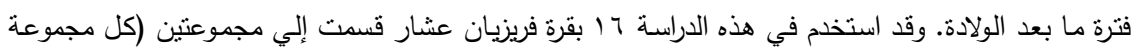

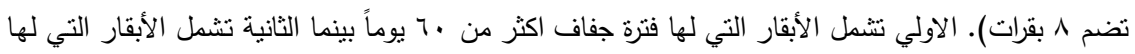
فترة جفاف أقل من ه؛ يوماً. التغيرات في وزن الجسم وقياسات الغدة اللبنية قدرت اسبوعياً بينما عينات نسيج

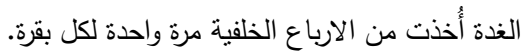

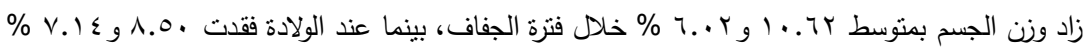

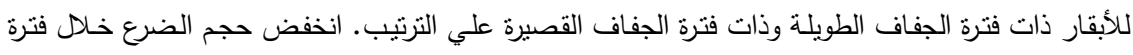

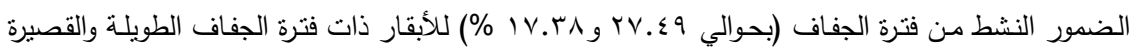

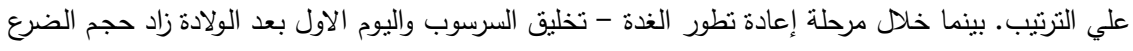

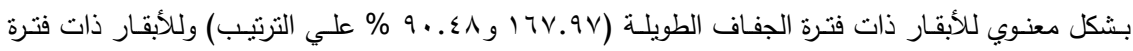

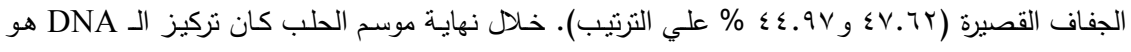

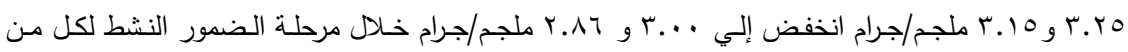
الأبقار ذات فترة الجفاف الطويلة والقصيرة علي الترتيب. خلال فترة نهاية موسم الحلب كان تركيز RNA هو البه

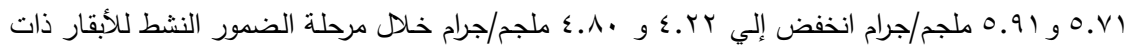

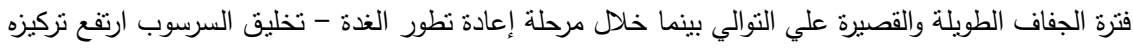

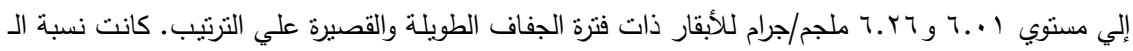

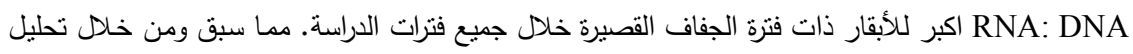

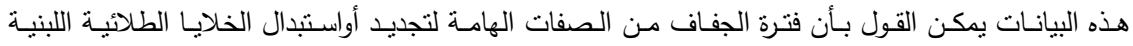

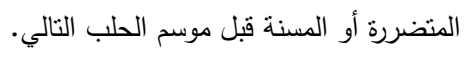

